\title{
Georgia's Rapid Expansion of Mosquito Surveillance in Response to Zika Virus
}

\author{
Chris Rustin*1, Deonte Martin ${ }^{1}$ and Rosmarie Kelly ${ }^{2}$ \\ 'Environmental Health Science, Georgia Southern University, Jiann-Ping Hsu College of Public Health, Statesboro, GA, USA; \\ ${ }^{2}$ Georgia Department of Public Health, Atlanta, GA, USA
}

\section{Objective}

To describe the Georgia Department of Public Health's (DPH) mosquito surveillance capacity before and after Zika virus was declared a public health emergency, review and compare mosquito surveillance results from 2015 to 2016, and evaluate the risk of autochthonous vector transmission of Zika virus based on 2016 surveillance data of Aedes aegypti and Aedes albopictus mosquitoes.

\section{Introduction}

Zika virus was declared an international public health emergency by the World Health Organization on February 1, 2016. With Georgia hosting the world's busiest international airport and a subtropical climate that can support the primary Zika virus vector, Aedes aegypti, and secondary vector, Aedes albopictus, the CDC designated Georgia as a high risk state for vector transmission. Faced with a lack of mosquito surveillance data to evaluate risk of autochthonous transmission and a few counties statewide that provide comprehensive mosquito control, the DPH rapidly scaled up a response. DPH updated existing mosquito surveillance and response plans targeted for West Nile Virus (WNV) and expanded capacity to areas that lacked previous surveillance targeting the Zika virus vector.

\section{Methods}

Mosquito surveillance data provided by DPH was analyzed for years 2015 and 2016 to date. The geographical distribution of counties conducting surveillance, total number and percentage by mosquito species collected in 2015 were compared to 2016 data. The distribution of counties conducting surveillance was mapped using ArcMap 10.4.1 for pre and post Zika response. Autochthonous vector transmission risk was evaluated based on the overall numbers and percentages of Aedes aegypti and Aedes albopictus mosquitoes collected for 2016 .

\section{Results}

In 2015, Georgia had 14 counties conducting mosquito surveillance, with a DPH entomologist providing direct surveillance in 4 of these counties. In 2016, DPH expanded surveillance capacity to 34 counties, a $142 \%$ increase, geographically dispersed across the State in urban and rural areas. A total of 76,052 mosquitoes were trapped and identified in 2015 compared to 91,261 mosquitoes trapped to date in 2016 , representing a $20 \%$ increase. A total of 37 mosquito species were identified in both years with Culex quinquefasciatus, Georgia's primary WNV vector, representing the highest percentage (2015-79.45\% and 2016-70.41\%) of mosquitoes trapped overall. In addition, Aedes aegypti represented only $0.108 \%$ and $0.007 \%$ of the total mosquitoes trapped respectively each year and was found in one county. Aedes albopictus represented only $1.50 \%$ and $1.82 \%$ of the total mosquitoes trapped respectively each year and was found in a majority of the counties conducting surveillance.

\section{Conclusions}

DPH was able to rapidly expand its surveillance capacity statewide by maximizing existing grant funds to hire new surveillance staff while also collaborating with academic institutions, military bases,
Georgia Mosquito Control Association, and local health departments to provide training and funding for surveillance and data sharing. This expanded surveillance network provided a clearer picture of the types of mosquitoes potentially exposing the public to mosquito-borne disease risks.

Historical data for the primary vector of Zika virus, Aedes aegypti has been isolated to just two counties in Georgia. Expanded surveillance in 2016 confirmed a low abundance of Aedes aegypti, suggesting the primary vector for Zika has been displaced by Aedes albopictus. This may suggest a reduced risk of autochthonous transmission of Zika virus in Georgia due to Aedes albopictus' affinity for feeding on both humans and animals. This should be interpreted with caution due to limitations in the data related to unstandardized reporting techniques for each county. DPH is working with all counties to improve the quality of data collected and reported and continues to educate the public on ways they can reduce their individual risk of mosquito bites, which in turn reduces the risk of other mosquito-borne diseases such as WNV.

In conclusion, DPH's response to Zika virus allowed it to rapidly increase its surveillance footprint and with new data, make sound public health decisions regarding mosquito-borne disease risks.

\section{Keywords}

Zika; Surveillance; Autochthonous transmission; Aedes aegypti; Aedes albopictus

\section{Acknowledgments}

The authors thank the Georgia Department of Public Health for providing data and consultation on this study.

\section{${ }^{*}$ Chris Rustin}

E-mail: rrustin@georgiasouthern.edu 